\title{
ADOPTION OF SUSTAINABLE HUMAN RESOURCES MANAGEMENT IN TURKISH CONTEXT: CHALLENGES AND SOLUTIONS
}

\author{
DOI: 10.17261/Pressacademia.2021.1428 \\ PAP- V.13-2021(17)-p.91-93
}

\section{Kubilayhan Goc}

Aksaray University, Department of Business Administration, Aksaray, Turkey.

Istanbul Technical University, PhD Candidate, Macka, Istanbul, Turkey.

gock@itu.edu.tr, ORCID: 0000-0002-7481-0837

\section{To cite this document}

Goc, K., (2021). Adoption of sustainable human resoruces management in Turkish context: challenges and solutions. PressAcademia Procedia (PAP), V.13, 91-93.

Permanent link to this document: http://doi.org/10.17261/Pressacademia.2021.1428

Copyright: Published by PressAcademia and limited licensed re-use rights only.

\section{ABSTRACT}

Purpose- As societies' environmental and social sensitivities increase, companies try to adapt themselves to this new expectation. Sustainability discussions also come into our lives in this direction. The Sustainable Human Resources Management approach, which aims to balance these sensitivities between humans, organizations, and the environment, has increasingly attracted the attention of researchers since the 2010s. Although companies are committed to this new understanding, they often cannot go beyond symbolic activities. In companies, managers come to the fore as the implementer/executor of sustainability. The primary purpose of this study is to investigate the difficulties faced by companies in adopting Sustainable Human Resources Management practices and develop solutions to encourage the adoption of this concept.

Methodology- In this study, data were collected online through open-ended questions from sustainability experts and senior executives of companies in the BIST Sustainability Index. Content analysis technique, one of the qualitative analysis methods, was used in the analysis of these data.

Findings- When the study's findings were evaluated, it was determined that the challenges for sustainability in the Turkish context generally stem from internal factors. Accordingly, the biggest obstacles to Sustainable HRM activities are the weak institutional structures of the companies, the lack of knowledge and skills of the managers, the economic and financial obstacles, and the lack of support from the leaders and managers.

Conclusion- As a result of this study, various suggestions have been presented to managers and companies to adopt and realize sustainable HRM in the Turkish context. Managers and experts made suggestions mainly for the internal context (94\%). However, the recommendations for the external context (6\%) were less on their agenda. Internal context recommendations focus more on HRM (48\%) and organizational level policies (32\%). The suggestions that stand out based on sub-themes are: - Increasing the awareness of HRM employees and senior management (14.3\%), - Determination of short/medium/long term sustainability strategy and holistic implementation of objectives (11.1\%)and regarding the role of HRM, "HRM should be goal setting, inclusive, transparent, and keep abreast of developments and process (11.1\%). When the study results are evaluated, Turkish companies should first prepare their internal processes to realize Sustainable HRM fully. In addition, according to the recommendations of the managers and experts regarding the external context, it is seen that the legal regulations will act as a driving factor in fulfilling the requirements of Sustainable HRM.

Keywords: Sustainability, human resources management, adoption, barries and solutions, Borsa İstanbul.

JEL Codes: Q56, 015, M50

\section{TÜRKIYE BAĞLAMINDA SÜRDÜRÜLEBILIR INSAN KAYNAKLARI YÖNETIMININ BENIMSENMESi:} ZORLUKLAR VE ÇÖZÜMLER

\section{ÖZET}

Amaç- Toplumların çevresel ve sosyal hassasiyetleri arttıkça, firmalar da kendilerini bu yeni beklentiye uyarlamaya çalışmaktadırlar. Öyle ki sürdürülebilirlik tartışmaları da bu doğrultuda hayatımıza girmektedir. İnsan, örgüt ve çevre arasındaki bu hassasiyetler üzerinde denge sağlamayı amaç edinen Sürdürülebilir İnsan Kaynakları Yönetimi anlayışı, 2010'lu yıllardan itibaren artan bir şekilde araştırmacıların ilgisini çekmeye başlamıştır. Firmalar kendilerini bu yeni anlayışa adamış olsalar da, çoğunlukla göstermelik faaliyetlerin ötesine gidememektedirler. Firmalarda yöneticiler, sürdürülebilirliğin uygulayıcısı/yürütücüsü olarak ön plana çıkmaktadır. Bu çalışmanın temel amacı, firmaların 
Sürdürülebilir İnsan Kaynakları Yönetimi uygulamalarını benimsenmesinde karşılaştıkları zorlukları araştırmak ve bu kavramın benimsenmesini teşvik etmek için çözüm önerileri geliştirmektir.

Yöntem- Bu çalışmada, BísT Sürdürülebilirlik endeksine dahil firmaların sürdürülebilirlikle ilgili uzmanlarından ve üst düzey yöneticilerinden online olarak açık uçlu sorular aracılığıyla veriler toplanmıştır. Bu verilerin analizinde ise, nitel analiz yöntemlerinden içerik analizi tekniği kullanılmıştır.

Bulgular- Araştırmanın bulguları değerlendirildiğinde, Türkiye bağlamında, sürdürülebilirliğin önündeki zorlukların genellikle firma içi unsurlardan kaynaklandığı tespit edilmiştir. Buna göre, Sürdürülebilir İKY faaliyetlerinin önündeki en büyük engeller: firmaların zayıf kurumsal yapıları, yöneticilerin bilgi ve yetenek eksikliği, ekonomik ve finansal engeller ve de lider ve yöneticilerin desteğinin eksikliği olarak sıralanmaktadır.

Sonuç- Bu çalışmanın sonucunda, Türkiye bağlamına ilişkin olarak, Sürdürülebilir İKY'nin benimsenmesi ve gerçekleştirilebilmesi için yönetici ve firmalara yönelik çeşitli öneriler sunulmuştur. Yönetici ve uzmanlar daha çok iç bağlama yönelik (\%94) önerilerde bulunmuşlardır. Dış bağlama yönelik öneriler ise (\%6) geri planda kalmıştır. İç bağlama yönelik öneriler daha çok íkY odaklı öneriler (\%48), ve örgüt düzeyi politikalara yönelik önerilerdir (\%32). Alt temalar bazında ise, IKY çalışanlarının ve üst yönetimin farkındalığının artırılması (\%14,3), kısa/orta/uzun vadeli sürdürülebilirlik stratejisinin belirlenmesi ve hedeflerin bütünsel olarak yürütülmesi (\%11,1) ve IKY'nin rolüne ilişkin olarak da "ikY hedef koyucu, kapsayıcı, şeffaf ve takip edici olmalı $(\% 11,1)$ önerileri göze çarpmaktadır. Çalışmanın sonuçları değerlendirildiğinde, Sürdürülebilir IKY'nin tam anlamıyla gerçekleştirilebilmesi için Türk firmaları öncelikle kendi içsel süreçlerini hazırlamalıdırlar. Bunun yanı sıra, yönetici ve uzmanların dışsal bağlama ilişkin önerilerine göre, yasal düzenlemelerin Sürdürülebilir İKY'nin gereklilikleri yerine getirmede itici bir faktör olarak işlev yapacağı görülmektedir.

Anahtar Kelimeler: Sürdürülebilirlik, insan kaynakları yönetimi, benimseme, engeller ve çözümler, Borsa İstanbul. JEL Kodları: Q56, 015, M50

\section{REFERENCES}

Dyllick, T., \& Hockerts, K. (2002). Beyond the business case for corporate sustainability. Business Strategy \& the Environment, 11(2), 130141. https://doi.org/10.1002/bse.323

Dyllick, T., \& Muff, K. (2016). Clarifying the Meaning of Sustainable Business: Introducing a Typology From Business-as-Usual to True Business Sustainability. Organization and Environment, 29(2), 156-174. https://doi.org/10.1177/1086026615575176

Elkington, J. (1997). Cannibals with Forks: The triple bottom line of 21st centuryThe triple bottom line of 21st century. Alternative Management Observatory, (April), 1-16. https://doi.org/http://doi.wiley.com/10.1002/tqem.3310080106

Emma, G.-M., \& Jennifer, M.-F. (2021). Is SDG reporting substantial or symbolic? An examination of controversial and environmentally sensitive industries. Journal of Cleaner Production, 298, 126781. https://doi.org/10.1016/j.jclepro.2021.126781

Esen, D., \& Süral Özer, P. (2020). Sustainable Human Resources Management (Hrm) a Study in Turkey Context and Developing a Sustainable Hrm Questionnaire. International Journal of Management Economics and Business, 16(3), 550-580.

Esen, D., \& Esen, M. (2018). Türkiye'de Yayınlanan Sürdürülebilirlik Raporlarının Sürdürülebilir İnsan Kaynakları Yönetimi Bağlamında İncelenmesi: Nitel Bir Araştırma. Uluslararası İktisadi ve İdari İncelemeler Dergisi, (17. Uík Özel Sayısı), 827-844.

Goc, K., \& Kusku, F. (2020). Sustainable human resources management from the language of reports. Pressacademia, 7(2), 95-115. https://doi.org/10.17261/pressacademia.2020.1202

GRI. (2013). G4 Sustainability Reporting Guidelines. Global Reporting Initiative. Amsterdam.

Guerci, M., Decramer, A., Van Waeyenberg, T., \& Aust, I. (2019). Moving Beyond the Link Between HRM and Economic Performance: A Study on the Individual Reactions of HR Managers and Professionals to Sustainable HRM. Journal of Business Ethics, 160(3), 783-800.

KPMG. (2020). The time has come: The KPMG Survey of Sustainability Reporting 2020.

Marimon, F., Alonso-Almeida, M. D. M., Rodríguez, M. D. P., \& Cortez Alejandro, K. A. (2012). The worldwide diffusion of the global reporting initiative: what is the point? Journal of Cleaner Production, 33, 132-144. https://doi.org/10.1016/j.jclepro.2012.04.017

Özgül, B., Bozkurt, S., \& Gürel, Y. (2020). Sürdürülebilirlik Perspektifinden Insan Kaynakları Uygulamalarının Incelenmesi: Görgül Bir Çalışma. Istanbul Business Research, (September 2019), 117-145. https://doi.org/10.26650/ibr.2020.49.0053

Özgül, B., \& Gürol, Y. (2019). Kurumsal Sürdürülebilirlikte Sürdürülebilir İnsan Kaynakları Yönetiminin Rolü Üzerine Bir İçerik Analizi. Doğuş Üniversitesi Dergisi, 20(1), 107-126.

Özutku, H., Çetinkaya, M., \& Ağca, V. (2015). İnsan Kaynakları Yönetimi Boyutuyla Kurumsal Sürdürülebilirlik: BisT Sürdürülebilirlik Endeksindeki Firmalar Üzerine Bir Araştırma. Süleyman Demirel Üniversitesi Iktisadi ve Idari Bilimler Fakültesi Dergisi, 20 (3), 55-72.

Purcell, J., \& Hutchinson, S. (2007). Front-line managers as agents in the HRM-performance causal chain: theory, analysis and evidence. Human Resource Management Journal, 17(1), 3-20. https://doi.org/10.1111/j.1748-8583.2007.00022.x

Rompa, I. (2011). Thesis - Explorative research on Sustainable Human Resource Management. University Amsterdam. Retrieved from 
http://www.innovatiefinwerk.nl/sites/innovatiefinwerk.nl/files/field/bijlage/sustainable_hrm.pdf

Seckin-Celik, T., \& Seckin-Halac, D. (2018). Sustainability Reporting on Labor Practices (pp. 331-351). https://doi.org/10.4018/978-1-52255757-9.ch017

Skouloudis, A., Evangelinos, K., \& Kourmousis, F. (2010). Assessing non-financial reports according to the Global Reporting Initiative guidelines: evidence from Greece. Journal of Cleaner Production, 18(5), 426-438. https://doi.org/10.1016/j.jclepro.2009.11.015

Vanderstraeten, A. (2015). The HRM Cockpit. An instrument for developing and evaluating sustainable HRM in an organization. Sustainable HRM and Employee Well-Being, Proceedings., 1-27.

Zaman, R., Farooq, M. B., Khalid, F., \& Mahmood, Z. (2021). Examining the extent of and determinants for sustainability assurance quality: The role of audit committees. Business Strategy and the Environment, 1-20. https://doi.org/10.1002/bse.2777 